\title{
A Planar Equiangular Spiral Antenna Array for the $\mathrm{V}$-/W-Band
}

\author{
Paul Tcheg, Kolawole D. Bello, David Pouhè \\ Reutlingen University of Applied Sciences, Alteburgstrasse 150, 72762 Reutlingen, Germany \\ paul.tcheg@reutlingen-university.de david.pouhe@reutlingen-university.de
}

\begin{abstract}
This work presents a spiral antenna array, which can be used in the V-and W-Band. An array equipped with Dolph-Chebychev coefficients is investigated to address issues related to the low gain and side lobe level of the radiating structure. The challenges encountered in this achievement are to provide an antenna that is not only good matched but also presents an appreciable effective bandwidth at the frequency bands of interest. Its radiation properties including the effective bandwidth and the gain are analyzed for the W-Band.
\end{abstract}

Index Terms-antenna array, circular polarization, effective frequency bandwidth, angle bandwidth.

\section{INTRODUCTION}

The V- and W-Bands include many interesting applications such as high-density wireless communication, millimeter-wave radar, satellite communications and amateur radio. Since the antenna is one of the most important devices in those engineering applications, some requirements including low profile, broadband properties and if desired, circular polarization have to be taken into consideration when designing this component. Due to significant atmospheric effects such as absorption and attenuation detected at the aforementioned frequency bands, particularly at $60 \mathrm{GHz}$ [1], a high gain antenna is also required.

One antenna candidate for those applications is the planar equiangular spiral. In earlier achieved studies [2], [3], it has been shown that the spiral antenna presents low profile as well as broadband features and by nature, it provides circular polarization. However, as any single patch element, it possesses relatively poor directivity/gain.

In this work, a planar equiangular spiral antenna (PESA) designed on substrate material along with its radiation performances is presented (Section II-A). In Section II-B a broadband tapered balun is developed. The performances of the PESA with the presence of balun are analyzed in Section IIC. Next to that, a 4x4-array equipped with DolphChebychev coefficients is investigated to address issues related to the low gain and side lobe level (SLL) of the radiating structure (Section III). Hurdles overcome throughout this work aimed at providing a PESA and an array that are not only good matched but also present an appreciable effective bandwidth at the frequency bands of interest.

All results are obtained using the simulation tool ANSYS Electronic Desktop 2016.2.

\section{THE STRUCTURE OF THE ANTENNA}

\section{A. Basic Antenna}

The design principles of spiral antennas are well known and can be found in early contributions [2], [3] and in any classical course book on antennas theory and design [4], [5], [6]. Due to space limitation, they will not be repeated here.

Fig. 1 shows the basic spiral antenna under consideration. Its expansion ratio $a$ is 0.3 which corresponds to an angle approximately equals to $73.3^{\circ}$. This choice of expansion value was motivated through the fact that it provides the best radiation characteristics with respect to return loss, input impedance and axial ratio when compared to antennas with arm growths of values 0.1 and 0.22 [7]. The arm length of the antenna is $5.5 \mathrm{~mm}$, which as per the well-known design equation (8) in [7] corresponds to an inner radius of $0.1 \mathrm{~mm}$ and an outer radius of $1.68 \mathrm{~mm}$.

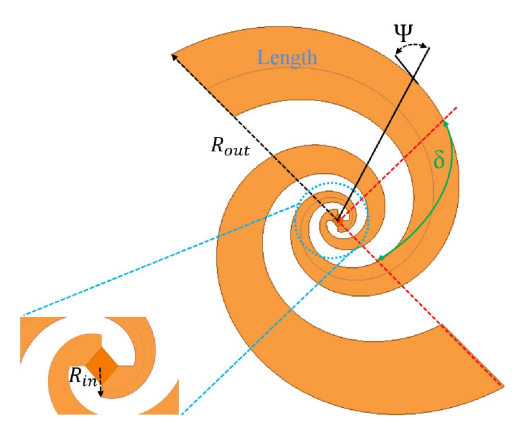

Fig. 1. Designed PESA. $\boldsymbol{a}=0.3 ; \delta=\pi / 2 ; R_{\text {out }}=1.68 \mathrm{~mm} ; R_{\text {in }}=0.1 \mathrm{~mm}$.

The antenna is designed on a substrate with a dielectric constant of $\varepsilon_{\mathrm{r}}=3$. The antenna shows a constant input impedance $Z_{\text {in }}=\eta /\left(2 \sqrt{\varepsilon_{\text {eff }}}\right) \approx 125 \Omega$ from $80 \mathrm{GHz}$ to 200 $\mathrm{GHz}$ and oscillates from $20 \mathrm{GHz}$ to $75 \mathrm{GHz}$ due to the reflections at the end of arms.

Fig. 3 depicts the return loss. As can be seen the impedance bandwidth of the antenna is large. It possesses a return loss bandwidth of more than $280 \mathrm{GHz}$ which includes the effective frequency bandwidth as can be observed by comparing Figs. 3 and 4 . Note that the effective frequency bandwidth, shortly called in the following effective bandwidth, is defined here as the band of frequencies where the antenna radiates a circularly polarized field. 


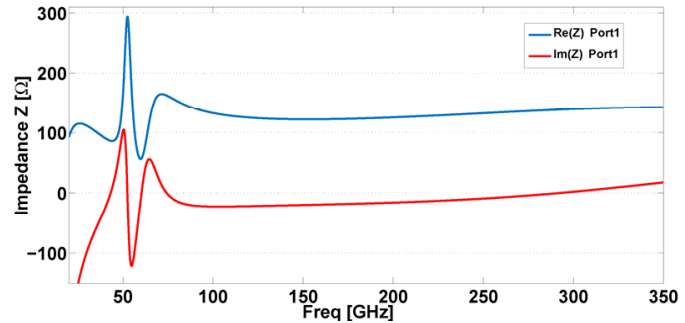

Fig. 2. Input impedance

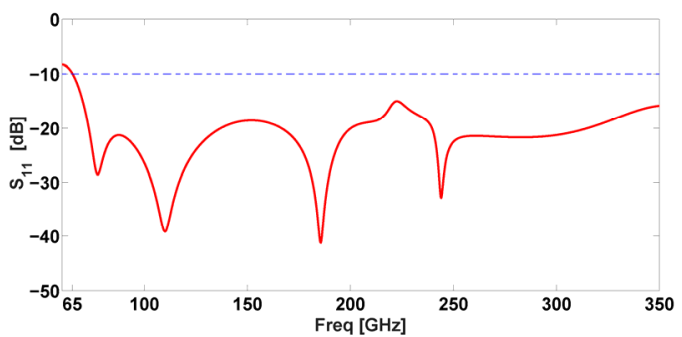

Fig. 3. Return Loss of the PESA.

Since the PESA is intended to radiate in the boresight direction, it may also be important to control the type of polarization of the radiated field and in circular polarization case, i.e. how broad the angle bandwidth is. All of the above is at a specific frequency. To that end, the frequency $83 \mathrm{GHz}$, which is included in the effective bandwidth, is chosen in order to observe the change of the polarization in the elevation direction by an azimuth angle $\phi=280^{\circ}$ and an angle sweep with $\vartheta$ as a variable is performed. Note that $\phi=280^{\circ}$ is just chosen as representative for all obtained results.

Fig. 5 depicts the change of the polarization of the PESA as a function of the elevation angle. At the observation point, the polarization remains circular for $70^{\circ}$. Thereby, the best axial ratio is recorded in the direction $20^{\circ}$ shifted from the plane perpendicular to the PESA.

Recall that the angle bandwidth is defined here as the band of angles where the antenna radiates a circularly polarized field in the boresight, respectively in the endfire direction (if desired) at a given frequency. The chosen frequency shall be within the effective bandwidth.

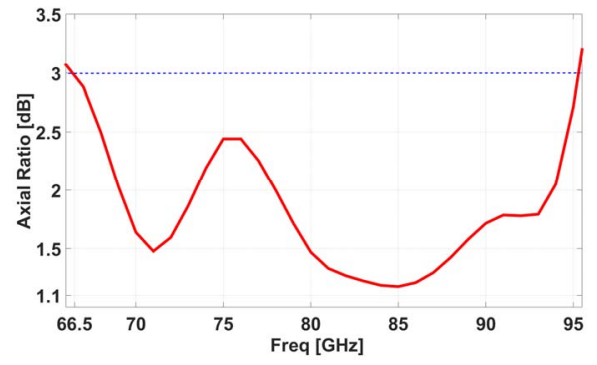

Fig. 4. Axial Ratio of the PESA. The effective bandwidth of the antenna is $27 \mathrm{GHz}$.

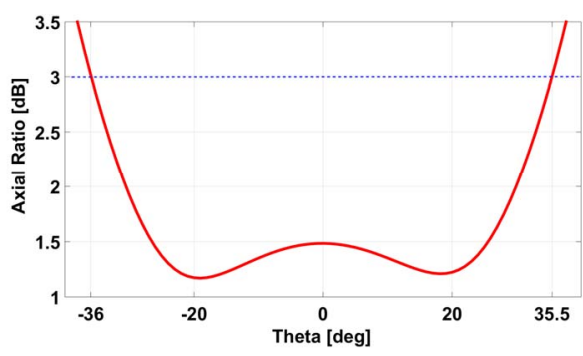

Fig. 5. Axial Ratio as function of the elevation angle showing the angle bandwidth.

\section{B. Design of broadband Balun}

To avoid any mismatch between the antenna and the transmission line on one side, and ensure a nearly perfect energy transfer from the generator to the radiating element and vice versa on the other side, an impedance transformer converting the $50 \Omega$ source impedance into the required $125 \Omega$ of the radiating element at the frequencies of interest is necessary.

The matching network designed in this work is a linear tapered one, whose design principles are widely developed in [8], [9] and will not be addressed here due to page limitation.

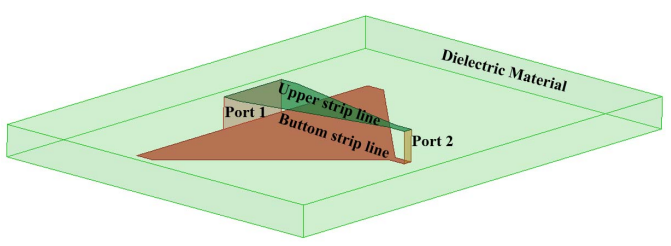

Fig. 6. Linear tapered balun.

The designed balun is graphed in Fig. 6. Its length, determined at the lowest simulation frequency of $20 \mathrm{GHz}$, is $L_{\text {Balun }}=3.7 \mathrm{~mm}$. The dimensions of the $50 \Omega$-side are $W_{\text {top }}=$ $0.64 \mathrm{~mm}, W_{\text {bottom }}=2.59 \mathrm{~mm}$ and the width of the $125 \Omega$-side is $W_{\text {strip }}=0.96 \mathrm{~mm}$. Since the width of the parallel strip line should match that of spiral arm, the substrate's height is $0.254 \mathrm{~mm}$. The balun shows VSWR values below 2 in the frequency range from 54.44 to $350 \mathrm{GHz}$ (Fig. 7).

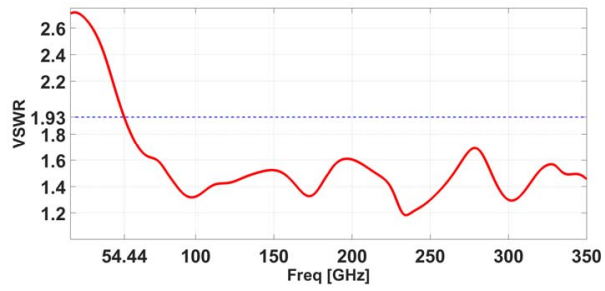

Fig. 7. VSWR of the designed linear tapered balun. 


\section{Analysis of the PESA with the Balun}

The radiating element and the balun were combined to obtain the structure presented in Fig. 8 and radiating characteristics of the PESA with balun were investigated.

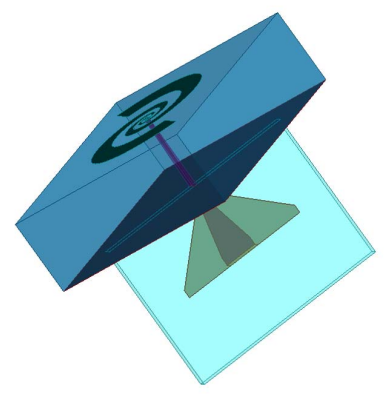

Fig. 8. Single Antenna-Balun Structure.

As shown in Fig. 9, the antenna-balun structure presents a good matching characteristic from 60.4 to $109.5 \mathrm{GHz}$. The noticed reduction of the bandwidth when compared to that of the radiating element and of the matching network is due to the abrupt transition from the vertical arrangement to the horizontal one at the line-antenna interface, leading thus to waves' reflections.

While analyzing the polarization of the structure, two observation points $\left[\phi=260^{\circ} ; \vartheta=20^{\circ}\right]$ and $\left[\phi=280^{\circ} ; \vartheta=20^{\circ}\right]$ presented good performances with a difference of $10 \mathrm{GHz}$. Due to the aforementioned reason in Section II-A, the achieved angle bandwidth is considered in Fig. 10.

At the observation angle $\phi=280^{\circ}$, a scanning beam of approximately $65^{\circ}$ from $\vartheta=-33.18^{\circ}$ to $\vartheta=30.47^{\circ}$, where the radiated field is circularly polarized, can be used. Fig. 12 shows the gain plotted over the frequency. Between $26 \mathrm{GHz}$ and $46 \mathrm{GHz}$ as well as between $66 \mathrm{GHz}$ and $92 \mathrm{GHz}$ the obtained gain is at least $4 \mathrm{~dB}$. A maximum gain of $7.48 \mathrm{~dB}$ is recorded at $71.18 \mathrm{GHz}$, where the polarization of the radiated field is also circular. A deterioration of the gain is noticed above $100 \mathrm{GHz}$, which corresponds to a part of the frequency band where the radiated field starts losing its circular polarization (see Fig. 11).

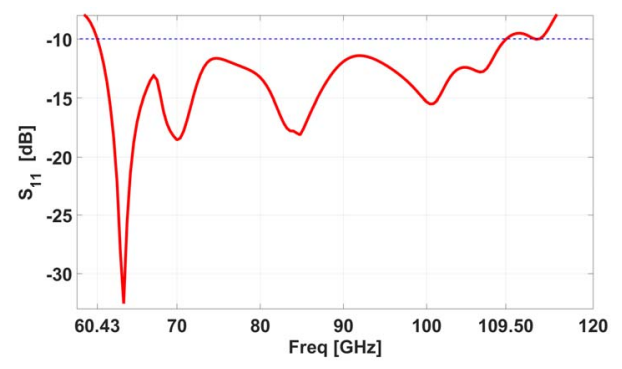

Fig. 9. Return Loss of the Single Antenna-Balun Structure.

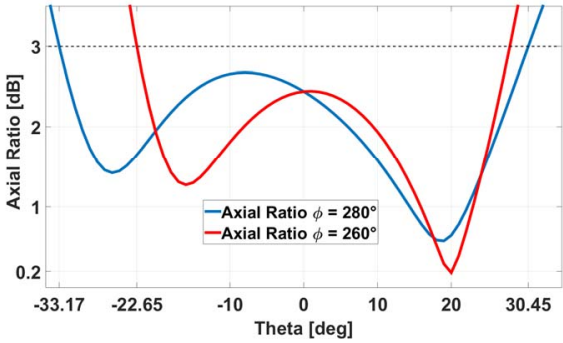

Fig. 10. Axial Ratio of the Single Antenna-Balun Structure as function of the elevation direction at azimuth angles $260^{\circ}$ and $280^{\circ}$.

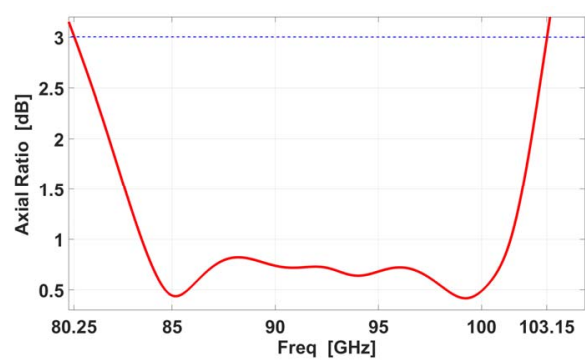

Fig. 11. Axial Ratio of the Single Antenna-Balun Structure as function of the frequency.

\section{THE ARRAY}

Usually, a single patch antenna element provides a relatively wide radiation pattern with a poor directivity. A PESA is no exception to that. In order to achieve a larger gain, radiating elements are disposed in a specific electrical and geometrical configuration, so that the whole electrical size of the antenna increases. In this section, a $4 \times 4$ PESA array is presented and its performances are evaluated. The PESA array is composed of 16 single PESAs, each implemented with its own balun. Since the designed PESA presents different dimensions when considering it in the $x y$ plane, the planar configuration will be constructed in two steps.

In the first step, a linear array consisting of 4 spirals aligned in row in the $y$-direction with respect to the width of the spiral is built. The distance between two consecutive radiating elements along the $y$-axis is calculated according to formula (1) [10]

$$
d_{y} \leq(1-1 / \mathrm{N})\left(\lambda_{0} /\left(1+\sin \vartheta_{S}\right)\right)
$$

where $\mathrm{N}$ represents the number of elements, $\vartheta_{S}$ the elevation angle and $\lambda_{0}$ the wavelength in vacuum. According to the formula right above, the value of $d_{y}$ should be less than 2.9 $\mathrm{mm}$ for $f_{0}=60 \mathrm{GHz}$ and choosing $\vartheta_{S}=15^{\circ}$. However, to avoid grating lobes, the distance $d_{y}$ should be chosen less than $2.5 \mathrm{~mm}$. Hence, $d_{y}$ is set equal to $2.2 \mathrm{~mm}$.

In the second step, 4 spirals are aligned again in row but this time along the $x$-axis and with respect to the height or the length of the spiral arm. Since the outer radius of one 
spiral is $1.68 \mathrm{~mm}$, the distance $d_{x}$ between two consecutive elements along the $\mathrm{x}$-axis is set to $3.3 \mathrm{~mm}$.

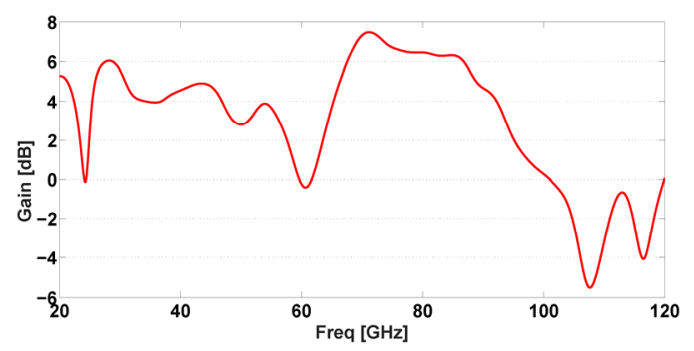

Fig. 12. Gain of the Single Antenna-Balun Structure over the frequency.

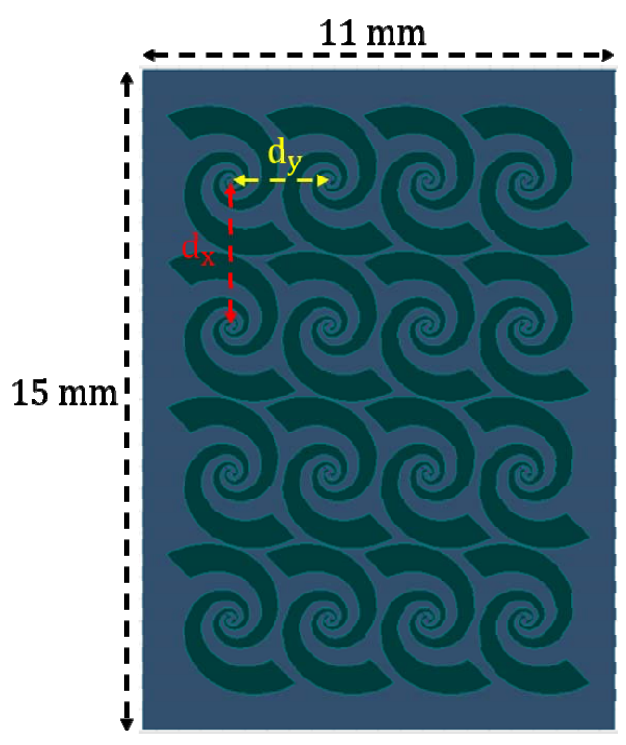

Fig. 13. 4x4 PESA-Array Layout.

Note that contrary to classical planar archimedean antennas, the distances $d_{x}$ and $d_{y}$ cannot be equal as the radius of equiangular spiral antenna increases with angle and thus leading to different distances along the $\mathrm{x}$ - and $\mathrm{y}$-axis. Moreover, the shape of the termination of the arm is along the radius vector.

An antenna synthesis was applied in order to achieve a Side Lobe Level (SLL) of $20 \mathrm{~dB}$. For this purpose, Chebyshevcoefficients were calculated according to the design procedure of Dolph-Chebyshev Array described in [4]. The determined coefficients $a_{1}=1$ and $a_{2}=2.33$ were applied as amplitude to the ports, as shown in table I.

\section{TABLE I. CHEBYSHEV-COEFFICIENTS RELATED TO THE} CORRESPONDING PORTS

\begin{tabular}{|c|c|c|c|}
\hline Port 1 & Port 5 & Port 9 & Port 13 \\
1 & 2.33 & 2.33 & 1 \\
\hline Port 2 & Port 6 & Port 10 & Port 14 \\
1 & 2.33 & 2.33 & 1 \\
\hline Port 3 & Port 7 & Port 11 & Port 15 \\
1 & 2.33 & 2.33 & 1 \\
\hline Port 4 & Port 8 & Port 12 & Port 16 \\
1 & 2.33 & 2.33 & 1 \\
\hline
\end{tabular}

The resulting array, shown in Fig. 13, is $15 \mathrm{~mm} \times 11 \mathrm{~mm}$ $\mathrm{x} 4.5 \mathrm{~mm}$ (considering the balun).

The return loss of the array is illustrated in Fig. 14. For a clear visualization of this parameter and since all recorded reflection-curves look almost similar, only $S_{22}$ and $S_{1515}$ are represented. The array achieves a return-loss-bandwidth of approximately $71 \mathrm{GHz}$ (from approximately 40 to $111 \mathrm{GHz}$ ) where a good impedance matching is performed. When comparing this bandwidth with the one of the single element, an increase of $20 \mathrm{GHz}$ was recorded. This is due to the constructive interference of propagating waves attenuating the reflections occurring at the line-antenna interface.

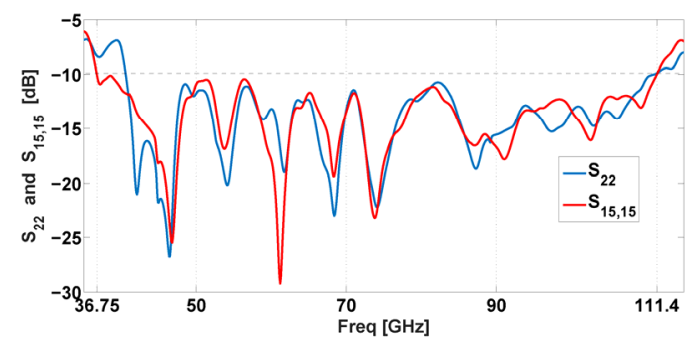

Fig. 14. Return Loss of the $4 \times 4$ PESA-Array Structure.

Within the frequency range from 40 to $111 \mathrm{GHz}$, the polarization behavior as function of the frequency as well as with respect to the elevation angle $\vartheta$ will next be investigated. Emphasis will however be put on the circular polarization.

Fig.14 depicts the behavior of the polarization over the frequency. It can be seen that the $4 \times 4$-array provides a circular polarization not only between 89.04 and $93.45 \mathrm{GHz}$ but also from 96.33 to $109.21 \mathrm{GHz}$. While analyzing this outcome, two important notes can be emphasized: First, the range of frequencies over which the array is circularly polarized is $5 \mathrm{GHz}$ smaller compared to that of the single antenna-balun structure. Secondly, there is a shift of the bandwidth towards higher frequencies. The reasons of these changes are mainly due to the rest of the energy that propagates towards the end of the spiral after the radiation occurring in the first active region of each spiral element [11]. On each single PESA, this residual energy interferes destructively with those of other PESAs. In addition, the reflected wave/energy from the spiral edge towards the feeding point possesses a reverse polarization compared to the polarization sense of the forward wave. This leads to the deterioration and in worst case to the cancelation of the circular polarization at lower frequencies, as well as the conservation of the polarization, in nature and sense, at higher frequencies.

Based on the obtained axial ratio over the frequency, the evaluation of the polarization with respect to the elevation angle is carried out. As applied in Sections II-A and II-C, a frequency within the bandwidth of circular polarization is chosen with the aim to analyze the angle bandwidth.

The polarization remains circular from $7.7^{\circ}$ to $32.01^{\circ}$ and from $-16.71^{\circ}$ to $-11.34^{\circ}$ which corresponds to a bandwidth 
of about $30^{\circ}$ (Fig. 16). Furthermore, it can be noticed that the angle of observation $\vartheta=10^{\circ}$ is included in the bandwidth. Indeed, the array radiates at $\vartheta=10^{\circ}$ a circularly polarized wave.

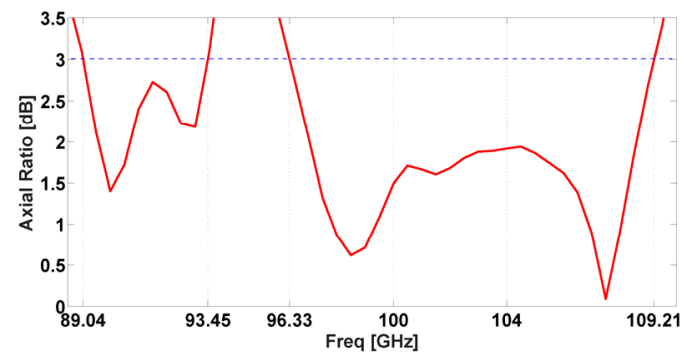

Fig. 15. Axial Ratio of the $4 \times 4$ PESA-Array Structure over frequency.

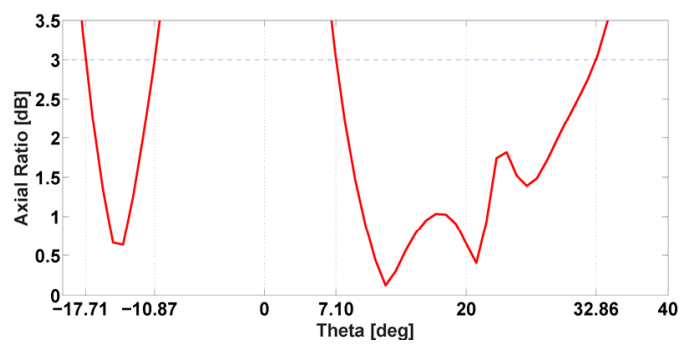

Fig. 16. Axial Ratio of the $4 \times 4$ PESA-Array Structure as function of the elevation direction at azimuth angle $290^{\circ}$ and frequency $104 \mathrm{GHz}$.

The use of an array antenna aims generally at improving the gain achieved by a single element. It is therefore worthwhile to take a look at this radiation parameter.

Fig. 17 shows the gain at the observation point $[\phi=$ $250^{\circ} ; \vartheta=10^{\circ}$. It remains at least by $10 \mathrm{~dB}$ over a large frequency band, which includes the range of frequencies over which the polarization is circular. A maximum gain of $15 \mathrm{~dB}$ is recorded at $74 \mathrm{GHz}$. A rapid decrease of the gain can be observed after the upper limit of the circular polarization bandwidth at around $109 \mathrm{GHz}$. Furthermore, a growth of the gain is visible when compared to that of the single element as expected. The gain of the array is twice that of the single element antenna. Although not presented here, it should be noted that for applications where the circular polarization is not desired, a maximum gain of 17.5 $\mathrm{dB}$ has been recorded at the observation point $\left[\phi=250^{\circ} ; \vartheta=\right.$ $0^{\circ}$.

Last but not least, Fig. 18 shows the radiation pattern. The SLL is as expected $20.12 \mathrm{~dB}$.

\section{CONCLUDING REMARKS}

This contribution addresses the design of a PESA, whereby the impedance transformer and also of a planar $4 \times 4$ PESAarray are designed in the V-/W-band. Some parameters such as the return loss and the gain are performed to evaluate the performances of the PESA-balun radiator as well as those of the $4 \times 4$-array. Dolph-Chebychev approach is applied to suppress grating lobes. The array

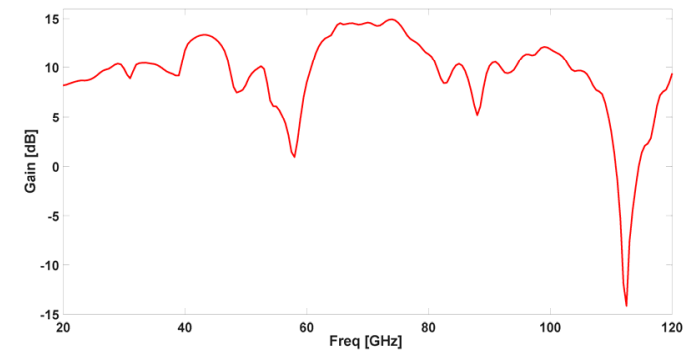

Fig. 17. Gain of the $4 \times 4$ PESA-Array Structure over the frequency.

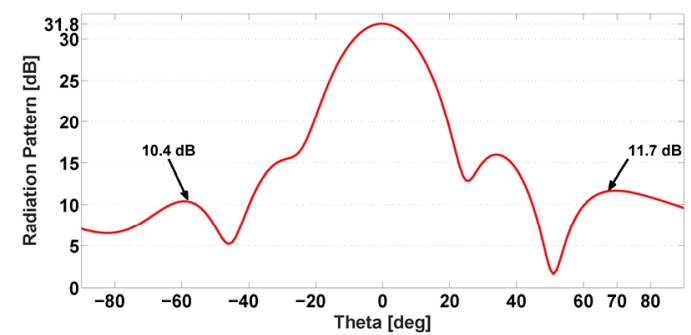

Fig. 18. Radiation Pattern of the $4 \times 4$ PESA-Array Structure over the elevation direction.

achieves a circular polarization bandwidth of $17 \mathrm{GHz}$ and a maximum gain of $15 \mathrm{~dB}$ at observation points included in the angle bandwidth. Since the focus is placed on the circular polarization over the frequency and also over the elevation angle, the good performances obtained in case of linear polarization aren't mentioned in this report. The next investigations will be carried out on the design of an appropriate feed network to provide apart from this plug-in solution, a fully compact and integrated solution.

\section{REFERENCES}

[1] ITU-R P.676-10, "Attenuation by Atmospheric Gases", Sept. 2013.

[2] V. H. Rumsey, "Frequency Independent Antennas", Eds. Academic Press, 1966, pp. 39-53.

[3] J. D. Dyson, "The Equiangular Spiral Antenna", IRE Trans. Antennas Propagation, Vol. AP-7, April 1959, pp. 181-187.

[4] C. A. Balanis, Antenna Theory: Analysis and Design, 3rd ed., New York: John Wiley \& Sons, 2005.

[5] R. C. Johnson, Antenna Engineering Handbook, 3rd ed., Mc GrawHill Professional, 1992, pp. 14-4-14-32.

[6] W. L. Stutzman and G. A. Thiele, Antenna Theory and Design, 2nd. Edition, John Wiley \& Sons, New York, 1998.

[7] B. Sirbu, T. Tekin, D. Pouhe, "Design and Simulation of an Equiangular' Spiral Antenna for Extremely High-Frequencies", The 8th European Conference on Antennas and Propagation (EuCAP 2014), April 6-11, 2014, pp. 3102-3106.

[8] J. W. Duncan, V. P. Minera, "100:1 bandwidth balun transformer", Proc. Inst. Elect. Eng., vol. 48, no 2, pp. 156-164, Feb. 1960.

[9] D. Pozar, Microwave Engineering, 4th ed., John Wiley \& Sons, 2005, pp. 246-267.

[10] K. W. Kark, Antennen und Strahlungsfelder, 4. Auflage, Vieweg Teubner Verlag, 2011, pp. 270-284.

[11] M. McFadden, W. R. Scott, "Analysis of the Equiangular Spiral Antenna on a Dielectric Substrate", IEEE Trans. Antennas \& Propagation, Vol. 55, No. 11, Nov. 2007, pp. 3163 - 3171. 